\title{
INFORME PRELIMINAR SOBRE EL RECORRIDO DEL VALLE DE CAÑETE \\ Louis Stumer
}

Como ya sabe la mayoría de ustedes, el proyecto arqueológico de la Costa Peruana de la Universidad Nacional Mayor de San Marcos, se efectúa en el año corriente, asimismo durante el año 1958-59, como un doble objetivo: el primero es un reconocimiento completo de los valles de la Costa desde el punto de vista de la preservación de sitios arqueológicos con valor científico y también de valor potencial para el turismo. Con este fin se están examinando cuidadosamente los daños actuales y potenciales causados por programas de irrigación y urbanización. El segundo fin es correlacionar las secuencias culturales de las tres regiones de la Costa Norte, Central y Sur, así como también el conocimiento del desarrollo cultural en las zonas de cada región.

Incidentalmente en este primer año, estamos subrayando más el recorrido general y se están reservando las excavaciones detalladas hasta el año entrante, en el que esperamos ofrecerles informes más detallados.

Específicamente en el valle de Cañete no existe mucho peligro de grandes daños en el futuro, porque las importantes obras de irrigación afectan solamente zonas en que no existen, afortunadamente, sitios arqueológicos.

Tampoco no hay en proyecto urbaniza- ciones. Se debe tratar en el Informe sobre el Plan General de los daños ya hechos, que voy a explicar con más detalle, sin embargo, podemos ver en la actualidad que este estado de cosas continúa, aunque no en forma drástica. Lo que posteriormente vemos en Cañete es, que en el pasado se ha observado la ley contra la destrucción de monumentos arqueológicos, pero sin conocimiento de lo que es un monumento arqueológico, creyendo que sólo consiste de arquitectura. Entonces encontramos que quedan en la mitad de los sembríos una gran cantidad de huacas cuyas zonas de viviendas y cementerios han desaparecido ya completamente, quedando nada más que fragmentos de cerámica y vestigios de artefactos en las acequias y en sus alrededores. En consecuencia en una revisión general se da un panorama d la arquitectura ceremonial o palaciega faltando en general el cuado de la vida diaria y de la muerte que es tan necesario para los fines del arqueólogo. El resultado de esta situación es también que cuando se construyen nuevas acequias sin especial cuidado se hacen inevitablemente daños a la arquitectura. Esto, está pasando en uno, dos o tres sitios, afortunadamente en lugares de menor importancia y valor.

Otro tipo de daño puesto en evidencia es el uso en el pasado de porciones de huacas como 
ladrilleras. Sin embargo, ya están casi todas abandonadas y no se sigue este tipo de destrucción. Ha habido en Cañete, hace más o menos 20 años, una gran pérdida. Debido a la geografía del Valle, la última obra de construcción de una gran acequia madre ha eliminado casi todos los cementerios que estaban ubicados en las faldas de los cerros. El resultado de esto es que casi todos los cementerios mencionados en la obra de Unanue ya están completamente cubiertos por algodonales, hasta el punto que es casi completamente imposible poder localizarlos.

Resumiendo casi todos los daños importantes ya han sido hechos y no creemos que exista mayor peligro en el futuro.

Con respecto al segundo objetivo debo informarles que el recorrido parece que ha probado el concepto de que la división entre la costa central y la costa sur se encuentra entre Cañete y Chincha. Hablo yo culturalmente y no estilísticamente. Todos los aspectos culturales de Cañete se reúnen con la costa central, y los de Chincha, como va a informarles oportunamente el Dr. Wallace, pertenecen a la Costa Sur. Sin embargo, tuvimos la suerte de encontrar una situación estilística un poco diferente, dándonos suficiente evidencia para la relación comparativa de los desarrollos respectivos. Entonces puedo decir que, en términos generales, han sido realizados los dos fines del recorrido de Cañete. Empero, naturalmente, existen sitios en los que se deben practicar excavaciones detalladas en el año entrante.

Yo concibo estas excavaciones como una serie que sirve para confirmar en cortes estratigráficos la secuencia cultural encontrada por nosotros en el recorrido; así mismo será necesario hacer cortes de pruebas para encontrar evidencias más detalladas de las culturas del valle así como especimenes dignos de las colecciones de la Universidad.

Ahora vamos a describir varios aspectos de la cultura del valle de Cañete encontrados en el recorrido mismo. Naturalmente, estas observaciones deben ser tomadas nada más que como preliminares, las que deben confirmarse solamente después de trabajar en el Museo con los materiales encontrados.
Arquitectura: Primero el plano general de los poblados (settlement pattern) en el valle de Cañete es algo diferente al que se encuentra en los otros valles de la Costa Central y esto es debido a la topografía. En esto hay 3 factores: $\left.1^{\circ}\right)$ existe un llano tan grande en la zona de la desembocadura del río que no había la necesidad de cuidar todo el terreno como en otros valles más pequeños; $2^{\circ}$ ) el ángulo de la subida de los cerros que rodean el valle es tan abrupto que no existen muchas posibilidades en la ceja de la zona regada; $3^{\circ}$ ) después del llano costeño empieza bruscamente el cañón del valle y, con excepciones, no existen en esa zona sitios adecuados para campos y pueblos. Entonces, la cantidad y el porcentaje de sitios encontrados en la zona regada misma es mucho mayor que la habitual.

En estos se nota la tendencia a edificar las construcciones en grupos o complejos y se constata en más de una ocasión la existencia de hasta 18 huacas en un complejo. Incidentalmente cito un ejemplar semejante en el valle del Rímac, el de Maringa o Aramburu. Actualmente, para subrayar esta tendencia, puedo decir que enumerado los complejos como sitios aislados hemos examinado unos cuarenta sitios; pero si se trata a cada huaca como un sitio individual, hemos examinado entonces más de 110 de ellas. Naturalmente, no hemos puesto un complejo en la categoría de complejo si no es por la evidencia de la cerámica o por los vestigios de conexiones arquitectónicas. Hablando en términos generales se nota en el valle de Cañete la misma con exactitud un desarrollo concreto de los materiales de construcción, lo mismo que en los otros valles de la costa central, en contraste con las secuencias detalladas encontradas por ejemplo, por Larco en la costa norte, y por Strong en la costa Sur. Pero al igual que en los otros valles de la costa Central. Existen factores generales y diagnósticos en el desarrollo de la arquitectura, tales como el uso de adobes hechos a mano, después varias combinaciones d estos y tapia, después tapia sola y por último, la aparición de adobes hachos a molde con o sin el uso de tapia adjunto. En asociación con el estilo cerámico más antiguo hasta el momento suficientemente identificado se nota el uso de adobes hechos a mano en forma de grano de maíz. Usando la terminología de la conferencia de Lima (1953) 
esa forma de adobito se atribuye a la época Formativa. Asociado con la época del Florecimiento regional se ve generalmente el uso de una forma de adobito típica de Cañete. Este es un adobito hecho a mano casi cúbico y de más o manos 18 centímetros de lado. Estos adobes se encuentran muy bien encuadrados o bien con ángulos imperfectos. En este último caso sin embargo, no sufre la construcción, porque con ellos se encuentra el uso experto de barro como cemento. En la última etapa de la época del Florecimiento regional los adobitos se encuentran generalmente cubiertos por una capa de arcilla fina, y dan la impresión de una construcción bien refinada.

También en esta etapa se nota el comienzo del uso general de la tapia con, o sin, el uso de los adobitos cúbicos. Se debe notar aquí que los sitios de la Época Formativa no presentan un aspecto imponente. Con la excepción del sitio de Cerro de Oro, publicado por Kroeber en 1937, los sitios de la época del Florecimiento Regional tampoco son ni muy altos ni masivos. Es decir, en otras palabras que no se nota en el valle de Cañete la presencia de sitios ceremoniales con el tamaño de los de Maranga, Juliana o Vista Alegre, del valle del Rímac. Sin embargo, el área misma de estos sitios y la cantidad de las construcciones que forman los complejos es bien grande. Entonces, la suma del trabajo encontrado en los sitios ceremoniales del Cañete podría haber sido más o menos la misma que la del Rímac. En la época Fusional, se nota la desaparición de los adobitos cúbicos y el uso general de una combinación de tapia y piedras del río en el valle de Cañete, porque se encuentran como cimentaciones, construcciones y también como adorno, puestas en forma geométricas en la superficie de la tapia. En las últimas etapas de la época Fusional y también en la época de Reinos y Confederaciones ya se ve el uso de la tapia en forma masiva, en la misma manera que en las huaquitas alrededor de Limatambo. Sigue el uso de la tapia en la época Imperial, pero se nota el comienzo del uso de adobes grandes rectangulares hechos a molde, cuyas dimensiones tienen un promedio de $45 \times 25 \times 8 \mathrm{~cm}$. Eso es en términos generales, el desarrollo del uso del adobe, pero debemos agregar unas palabras sobre el uso de la piedra. Además del uso de piedras del río que mencioné anteriormente, se encuentra tam- bién arquitectura de piedra en el único sitio que hemos encontrado en Cañete, que sospecho ya es precerámico. Más tarde se nota en el sitio de Cerro de Oro la presencia de unas murallas de piedra, muy bien construida, debajo de una construcción de adobes cúbicos. Ambos casos necesitan más investigación. Por último se nota la presencia de construcciones suplementarias muy bien hechas de piedra en sitios de la época Imperial.

En conclusión, el uso de la piedra no ha alcanzado gran importancia en la arquitectura de Cañete y también parece que sigue, en términos generales, el mismo desarrollo que en el valle del Rímac con su uso de piedras de sitios de gran antigüedad y después su reaparición en las últimas épocas. Hay unos sitios atípicos en cañete que se deben mencionar. El primero es el sitio Cerro de Oro, con sus numerosas construcciones y tumbas de la época Florecimiento Regional y sus intrusiones en la época Reinos y Confederaciones. Cerro de Oro es importante de mencionar porque lamentablemente el Dr. Kroeber, en el año 1925, no tuvo suficiente tiempo para una investigación completa de un sitio tan grande y extenso y ha dado por lo tanto en su informe la impresión de que s trata de una necrópoli inmensa, dividida más o menos por mitad entre el Cañete Medio y el Cañete Último.

Actualmente desde el punto de vista de las construcciones el sitio es aproximadamente en un $90 \%$ Cañete Medio o sea Reinos y Confederaciones, y se encuentran toda clase de restos arqueológicos del mismo, tales como construcciones, tumbas, basurales, hornos, etc. Tal vez también la práctica en la época Formativa y las del Florecimiento Regional de la costa central, de enterrar en pequeños grupos de todas las partes de un sitio de construcciones y no en cementerios concentradas como en épocas más tardías, ha dado a Kroeber una falsa impresión, porque se encuentra esa práctica por todo lado en Cerro de Oro. Las intrusiones en el Cañete Medio se limitan a unas adiciones a la arquitectura del Cañete Medio al lado Oeste del sitio, asimismo en un gran cementerio de este sitio, y, de vez en cuando, tumbas intrusitas por la falda sur del cerro. Las colecciones de fragmentos de la superficie muestran nada más que el estilo Cañete Medio excepto en las 
2 zonas ya mencionadas. Otro sitio atípico es la fortaleza de Ungará, con su uso ornamental de tapia dentro de las paredes protectoras. El examen superficial indica que todo pertenece al Incanato, tal vez dando apoyo a las referencias de los cronistas a la resistencia de la confederación tan grande y tan poderosa? Especialmente como la fortaleza está ubicada en el sitio más estratégico del valle para maniobras militares y el control del agua del río. Otro sitio atípico es la fortaleza de Chancarí ubicada en la mitad del valle, lo que podría haber sido la fortaleza de las Confederación Huayco, pero debido a la pequeña cantidad de fragmentos de diagnóstico encontrados, esa conclusión tiene que esperar que se realicen más trabajos.

Cerámica.- La existencia de fragmentos indica la presencia en la época Formativa de una cultura Chavinoide de la costa, semejante a la de Ancón, Supe, al norte, y de Jaguar pronta a ser descrita por el Sr. Lanning al Sur. Pero para establecer una secuencia cultural este estilo necesita y merece mucha más investigación. Desde luego en la hacienda La Quebrada, hemos encontrado lo que parece ser dos etapas de la siguiente cultura Formativa.

La primera etapa de esta cultura, que el autor está llamando tentativamente "La Quebrada”, se reúne estilísticamente con las culturas Paracas-Necrópolis y el nuevo Proto-Nazca de Strong, pero, al mismo tiempo, tecnológicamente hablando, es sencillamente de la costa central. Es decir, que la cerámica misma, y las formas de la misma, nunca pueden confundirse con una cerámica sureña. Se nota la presencia del diseño en patrón bruñido y del negativo junto con el policromado pulido con incisiones. Los colores usados en el policromado son blanco, negro y rojo borgoña. Todavía no se ha notado el gris y el amarillo que ha encontrado Strong en los valles de Nazca. De acuerdo con la pequeña muestra que tenemos hasta el momento se pueden notar formas sencillas de platos y cuencos chicos. También se encuentran antaras de arcilla fina bien pulida con encoge de rojo borgoña distintivamente de la manera Nazca. Como nota incidental, Cañete es lo más al norte en que hemos visto este tipo de antara. En los valles más al norte hay antaras decoradas, o con un engobe pero no aparecen con el rojo oscuro sureño. La segunda etapa del estilo La Quebrada, o sea "La Quebrada 2" exhibe también la influencia estilística de Nazca. Ya ha desaparecido la cerámica tipo Proto-nazca, así mismo todas las manifestaciones de Paracas-Necrópolis, con la excepción tipo marrón claro por fuera, y negro pulido por dentro. Este, sin las incisiones y sin el patrón, bruñido de la etapa anterior. Ahora se nota el comienza de una forma típicamente Cañete, o sea un plato con orilla angular. El desarrollo de esta forma es muy interesante, pero lamentablemente no tenemos suficiente tiempo ni espacio en este informe para describirla en detalle. Regresando a los platos de la Quebrada 2, usualmente se nota la presencia de una banda de color rojo oscuro rodeando la orilla; el resto del plato interior y exterior se encuentra bien pulido pero sin engobe ni dibujos. Parece que se puede relacionar cronológicamente la Quebrada 2 con nazca "A", debido a la presencia de dibujos típicos, y también hechos a la manera nazca, de peces y pajaritos. No se notan influencias de otras regiones en el resto de los dibujos encontrados; son sencillamente locales. El siguiente estilo cerámico es lo que ha llamado Kroeber Cañete Medio, pero estor usando, según las normas del a Conferencia de Lima, el término "Cerro de Oro" del sitio tipo. Este también parece que existe en dos etapas, pero tenemos que confirmar estas teorías con las excavaciones. Sin embargo, se encuentra en el tipo I la misma forma del plato que en La Quebrada 2, así mismo influencias del Nazca "B" mientras que en el segundo tipo ya no hay esos platos (son todos del tipo descrito e ilustrado por Kroeber, lámina 71 y 72) y las influencias en el dibujo son del Ayacucho policromo de Bennett, o sea el nazca "Y" de Kroeber. No hay necesidad de describir en detalle aquí el estilo Cerro de Oro, no hemos descubierto diferencias mayores en nuestras muestras a las ilustraciones de Kroeber. Solamente quisiera notar el comienzo en el estilo del valle de Cañete. Mi observación personal e que Cañete es la fuente de origen de esta forma porque el Dr. Wallace me ha informado que no existe en Chincha, y no ha visto en las excavaciones Strong en Nazca y no aparece al norte de Cañete hasta la época Fusional en Cerro de Oro 1 se nota por primera vez la presencia de influencias estilísticas no sureñas. Estas son motivos encontrados en el Playa Grande Tardío y también en Maranga. Son los elementos derivados de tejidos. 
Llegando a la época Fusional, tengo que informarles a riesgo de pecar de escéptico que no hemos encontrado evidencias Tiahuanacoides. Los estilos de la época Fusional se distinguen solamente por la desaparición de los motivos y las formas de la época Florecimiento Regional, así mismo por la decadencia técnica tan típica de la época y por la aparición de las formas perfeccionadas en la época Reinos y Confederaciones. Vamos a tener mucha dificultad con la declinación de las etapas cerámicas de la época Fusional en Cañete. Lo único que me permite poner esos estilos en la época Fusional es la presencia muy de vez en cuando de motivos decadentes Tiahuanacoides. Tan decadente que son casi indistinguibles.

Así mismo, en el Cerro de Oro 1, empieza una cosa completamente típica de la cerámica pretiahuanacoide de Cañete. Eso es la desaparición del rojo de borgoña nazqueño reemplazándose por un color francamente morado. Este morado, frecuentemente en combinación con un blanco algo verde, es casi marca registrada de Cañete. En vasijas cerradas comas ollas o botellas, aparecen en bandas grandes o por el gollete; en vasijas abiertas como platos o cuencos aparece como engobe interior. Debo decir que el resultado que nunca va a ser una de las glorias de la cerámica antigua peruana.

No necesitamos pasar mucho tiempo con la cerámica de la época. Reinos y Confederaciones porque esta es sencillamente el estilo Cañete Último tan bien descrito por Kroeber, por esto estamos usando tentativamente el término Huayco. Solamente tengo que hacer dos observaciones: la primera, es que nunca he visto en la cerámica peruana un uso tan grande de cerámica negra. El porcentaje de cerámica roja es minúsculo en cualquier tipo de vasija, sea utilitario o ceremonial. Se encuentra también la cerámica negra en cualquier tamaño; desde miniaturas de tres centímetros de altura hasta tinajones. La otra observación es que podemos relacionar el estilo muy bien con el estilo Chincha de la época Reinos y Confederaciones, según el uso de las formas Cañete en ejemplares chichanos encontrados por Wallace. Incidentalmente debo indicar que hemos encontrado en nuestras muestras del estilo, solamente un fragmento del que se podría decir que tiene algo de influencia chinchana.
La época Imperial exhibe más información cuzqueña y menos ejemplares de estilos locales o sea en los estilos llamados por Strong "Inca Asociados" como en el caso de los valles más al norte. Estoy hablando de dibujos en este caso, porque como en los otros valles de la costa durante el Incanato se encuentra dibujos serranos sobre formas locales. También las formas incaicas aparecen en porcentaje normal. Todavía no he sido aislado con certeza algo colonial.

Con este breve resumen pasaremos a los tejidos y otros artefactos.

En términos generales, los tejidos del valle de Cañete no sufren en comparación con los otros valles de la costa central así como tampoco son mucho mejores. Faltando la influencia tiahuanacoide, durante cuya época se notan tejidos tan lindos en las zonas afectadas, se puede decir de acuerdo con nuestras investigaciones que los tejidos de la época Florecimiento Regional son los mejores que hemos encontrado. El tipo está muy bien ilustrado por Kroeber en su lámina 89, pero se tienen que ver los tejidos mismos para apreciar la ejecución, la finura de su hilo de algodón y la brillantez de sus colores, hay también tapicería excelente. Junto con ellos se debe notar la presencia de tejidos de pluma de calidad excepcional. Todavía no hemos encontrado más que tejidos bien sencillos de la época Formativa pero su tipo técnicamente son buenos y comparables a los tejidos de la misma época en otras partes de la costa central.

En la época Fusional se nota una decadencia notable en la finura de los tejidos y el inicio algo característico de los tejidos de las épocas tardías. Esto es, el uso de doble urdimbre o trama y de vez en cuando de ambos. Se nota en los tejidos de esas épocas el uso general de rayos en marrón blanco y la casi sola diferencia entre grupos de estos tejidos está en el grado de oscuridad del marrón. Menos acostumbrados es el uso de rayas en azul claro, casi turquesa y blanco. Debo anotar aquí que estoy tratando de tejidos de algodón; el uso de la lana no es de ninguna manera común antes de la época Imperial. Se nota también en las épocas tardías el empleo de todas las técnicas halladas en los otros valles costeños para la misma época. 
En la época Imperial se encuentra por la primera vez el uso general de la lana según las normas de los tejidos incaicos costeños. Para hablar en más detalles de los tejidos necesitaremos hacer mucho más análisis en el laboratorio.

Hablando ahora de otros artefactos se ha confirmado en el recorrido del Cañete la presencia de todos los tipos de artefactos ya mencionados e ilustrados por Kroeber. Solamente quisiera mencionar algunas peculiaridades. Una es la presencia Florecimiento Regional de una cantidad notable de discos de cerámica, usualmente bien decorados con diámetro de un promedio de $12 \mathrm{~cm}$ aproximadamente. Ellos tienen huecos en su centro de aproximadamente $6 \mathrm{~cm}$. Solamente voy a repetir las palabras del Dr. Willey con respecto a un artefacto que se encontró en Supe: "el uso de este artefacto es problemático". También el resto de la época Florecimiento Regional hemos encontrado una variedad de artefactos que voy a tratar de describir a pesar de mi español. Tienen estos un largo de más o menos $9 \mathrm{~cm}$ y consisten en una saeta delgada de madera o hueso muy bien hecho, con una punta redondeada, y al otro lado se nota un tapón cilíndrico de unos 2 1/2 $\mathrm{cm}$ de diámetro, también hecho de hueso o de madera. Los artefactos han sido encontrados en cualquier combinación de los 2 materiales. Hemos pasado mucho tiempo antes de poder encontrar uno de estos artefactos in situ. Son talas para mates.

Incidentalmente, el uso de tapas tan bien hechas para mates sugeriría la presencia de una cantidad notable de mates pirograbados. Este es el caso.

La tercera cosa que quiero anotar es la cerámica de los piruros de la época Reinos y Confederaciones o sea el estilo Huayco. Al igual que su otra cerámica empleando exclusivamente cerámica negra, extraordinariamente bien pulida.

En la metalurgia, confírmense también las observaciones de Kroeber. En la época Pre-Fusionales se encuentra el uso oro y cobre en porcentaje normal. En las épocas post-Fusionales aparece plata y también quizá bronce, pero en cuanto a esto debe esperarse el resultado de análisis. Solamente es notable la cantidad de metales en las épocas tardías. Aparecen no solamente en los entierros ricos sino hasta en los de los pobres. Aparecen también joyería y herramientas.

Unas palabras sobre los enterramientos. Las únicas tumbas investigadas pertenecieron a las culturas Cerro de Oro y Huayco respectivamente. Se nota en Cerro de Oro tumbas cuadrangulares de adobitos cúbicos adosadas a las faldas del cerro y esto ocasiona una gran diferencia en la profundidad al lado alto de 2 m y más, y al lado bajo de nada más que 50 $\mathrm{cm}$. Los cadáveres son enterrados sentados, no en forma de fardo, solamente con sus propios vestidos y exhiben consistentemente una deformación craneana de forma frontal característica. Hay evidencia en esta época de tumbas grandes e importantes de personajes, construidas con adobitos cúbicos en la misma manera pero usando para 2 ángulos de cuadrángulo un rincón exterior de una construcción. Esas se encuentran encima de Cerro de Oro y parece que tuvieron techos muy bien construidos de adobitos con capa de arcilla fina. La evidencia dejada por los huaqueros indica que eran tumbas múltiples de tres o cuatro personajes y un igual número de servidores. Se habla por los naturales de la riqueza de oro y encontrado en estos formas de tumbas cosa que no desestimo yo según la construcción de las tumbas y por la calidad de los vestigios de tejidos y otras cosas allí dejadas. La conservación en tumbas tipo Cerro de Oro es uniformemente buena.

Las tumbas de la cultura Huayco son en su mayoría circulares, usualmente con un diámetro de 60 a $85 \mathrm{~cm}$ y con una profundidad de más o menos $1 \frac{1}{1 / 2} \mathrm{~m}$. Naturalmente hay diferencias normales en estas dimensiones. Se nota la presencia de techos de madera y cañitas y también de caña brava y paja. Los enterramientos se encuentran en forma de fardo con envoltura exterior de esteras. Hay ausencia completa de deformación y el único entierro extraordinario que hemos encontrado era uno correspondiente a un enano.

Se encuentra los tinajones y la cerámica utilitaria sueltos en las tumbas; las vasijas pequeñas y ceremoniales, así mismo los artefactos chicos, se encuentran dentro del fardo. Ca- 
nastas con materiales de costura son usuales. Usualmente los enterramientos de este estilo se encuentran debajo de una capa de caliche y este en combinación con los techos mencionados más arriba aseguran una conservación siempre excelente.

Partiendo del principio de que es sólo una conclusión preliminar y el resultado solamente del recorrido, el autor estima que la secuencia cultural de Cañete parece ser lo siguiente: Época Formativa, Chavinoide y La Quebrada 1; Época de Florecimiento Regional, La Quebrada 2, Cerro de Oro 1 y parte de Cerro de Oro 2; Fusional, parte de Cerro de Oro 2 y estilos locales todavía no nombrados; Reinos y Confederaciones, Huayco, Imperial, Inca Cañete o Inca Asociado. Al relacionar esta secuencia con las secuencias de la costa sur tendremos que examinar un poco más los estilos de la época Fusional para concretar la idea. El autor espera que esto podrá hacerse con los futuros recorridos del valle de Pisco. El resto parece ser bien sencillo y los remite a las semejanzas arriba citadas.

En conclusión, no he notado en lo referente a la revolución urbana diferencias notables en el valle de Cañete, tal vez con la excepción de la forma diferente de los centros ceremoniales de la época de Florecimiento Regional y de la ausencia de un "urban elite center". Pero en éste existe un sitio que denomino yo como "provincial elite" que autoridades con más conocimientos que yo, como los Drs. Schaedel y Willey, tal vez van a llamar "urban elite". En otras palabras, ese sitio casi cumple los requisitos para ambos tipos de sitio; el nombramiento es más o menos de acuerdo al gusto. El desarrollo urbano publicado por Schaedel en 1951 y para los centros norteños y por Willey y yo en 1954 para los valles de Virú y Rímac, respectivamente, tiene validez para el Cañete también.

Una cosa que quisiera subrayar es la dualidad estilística del valle referente a las influencias estilísticas del valle referente a las influencias adoptadas allá. No he tenido suficiente espacio en este informa para describirlo en detalle, pero es importante hacer notar que antes de la época Fusional en 90\% de las influencias extranjeras en la cerámica vinieron del sur; después del Fusional, casi el 100\% proviene del norte. La explicación del fenómeno necesitará indudablemente mucho más estudio. Otra cosa interesante es la impresión de aislamiento comparativo que nos da el valle. No obstante, la presencia de influencias estilísticas en la cerámica, en cantidad suficiente para relacionar los estilos de Cañete con los estilos vecinos, no existe en gran porcentaje. En cuanto a otras manifestaciones culturales extranjeras son más bien conspicuas por su ausencia, la arquitectura es casi completamente local hasta el Incanato, se aprecia la ausencia de lana hasta la misma época; la ausencia de Tiahuanacoide, y el acento sobre las formas locales en cualquier época de la cerámica. Se recibe la impresión de que el valle de Cañete con su abundancia de agua y su consecuente riqueza agrícola no necesitaba mucho contacto económico con sus vecinos y como consecuencia de esto no ha sido el depósito para muchas influencias culturales foráneas y que ha sido más de lo que ha recibido. Esta presunción también puede explicar la resistencia evidente a invasiones del exterior.

Debo y quiero en este momento agradecer muchísimo la ayuda de los alumnos, Srta. Rosa Fung Pineda y Sres. Luis Lumbreras y Ramiro Matos de la Universidad de San Marcos de Lima. 